\title{
Spectroscopic and photometric study of the bright X-ray cluster A1300
}

\author{
M. Pierre ${ }^{1}$, J. Oukbir ${ }^{1}$, D. Dubreuil ${ }^{1}$, G. Soucail ${ }^{2}$, J.-L. Sauvageot ${ }^{1}$, and Y. Mellier ${ }^{2,3}$ \\ 1 CEA Saclay DSM/DAPNIA, Service d'Astrophysique, F-91191 Gif sur Yvette, France \\ 2 Observatoire Midi-Pyrénées, Laboratoire d'Astrophysique de Toulouse, UMR 5572, 14 Avenue E. Belin, F-31400 Toulouse, \\ France \\ ${ }^{3}$ Institut d'Astrophysique, 98bis Bd. Arago, F-75014 Paris, France
}

Received July 22; accepted November 6, 1996

\begin{abstract}
We present the first results of an optical follow-up of X-ray bright clusters of galaxies located between $0.15 \leq z \leq 0.31: B$ and $R$ photometry as well as medium resolution spectroscopy of the galaxies. The cluster studied here - A1300 - is found to have a redshift of 0.3072 and a velocity dispersion of $1210 \mathrm{~km} / \mathrm{s}$, based on 52 cluster members. Structures are observed in the galaxy number counts but the velocity histogramme shows no significant departure from a Gaussian distribution ${ }^{1}$.
\end{abstract}

Key words: galaxies: clusters: individual: Abell 1300 $\mathrm{X}$-rays: galaxies — galaxies: redshifts

\section{Introduction}

Young rich clusters have very important cosmological implications since they are supposed to originate from the rare initial density fluctuations of high amplitude. In this general context, we have initiated a high resolution multiwavelength campaign (IR, radio, optical, X-ray) in order to study in detail the properties of distant bright X-ray clusters newly discovered in the ROSAT All-Sky-Survey (RASS), especially in order to better understand their dynamical state (Pierre et al. 1994a). We have selected a set of $\sim 10$ clusters, having X-ray luminosities greater than $10^{44} \mathrm{erg} / \mathrm{s}$ and thus expected to be massive. This implies a high density for the intra-cluster gas and most likely, a deep gravitational potential. The objects are located in the southern hemisphere between $10 h \leq R A \leq 14 h$ within

Send offprint requests to: M. Pierre mpierre@ariane.saclay.cea.fr

* Based on observations collected at the Canada-FranceHawaii Telescope at Mauna Kea, Hawaii, U.S.A.

1 Table 3 (4) is only (also) available in electronic form at the CDS via anonymous ftp to cdsarc.u-strasbg.fr (130.79.128.5) or via http://cdsweb.u-strasbg.fr/Abstract.html an area covering some 1700 square degrees. The redshifts cover the range $z=0.15-0.31$, which is ideal for mapping the whole cluster extent within reasonable exposure times. It will also be possible to tackle question related to cluster evolution by comparison with lower redshift observations. The cluster of galaxies A1300 is one of the priority target of the programme and we present here the first results of the detailed optical follow-up concerning this object.

A1300 is described as a richness class 1 object by Abell et al. (1989) and had not drawn specific attention from the community so far. It was observed for the first time in X-ray during the RASS and notified as such during a subsequent identification campaign at the ESO $3.6 \mathrm{~m}$ Telescope (Pierre et al. 1994b). These optical observations have shown that the cluster is quite rich, very extended, has a redshift of $\sim 0.306$ and suggested a high velocity dispersion. The RASS X-ray image is clearly extended, approximately in the SN direction (Pierre et al. 1994b). Preliminary analysis of a subsequent deep PSPC ROSAT pointing shows a distinct clump at the northern end, some $3^{\prime}$ from the cluster center, corresponding to a conspicuous enhancement of the galaxy density. The X-ray luminosity appeared to be high, $\sim 10^{45} \mathrm{erg} / \mathrm{s}$ in the ROSAT band, and hard. All of this indicate that A1300 is quite an interesting object - probably exceptional -, one of the few $z \geq 0.3$ clusters having a high X-ray luminosity.

Throughout the paper we assume a Hubble constant of $50 \mathrm{~km} / \mathrm{s} / \mathrm{Mpc}$ and $q_{0}=1 / 2$.

\section{Observations}

Four bright X-ray distant clusters detected in the RASS - including A1300 - have been observed during a 4 night run at the $3.6 \mathrm{~m}$ CFH Telescope in May 1993. For A1300, medium deep images were obtained in the $B$ and $R$ bands as well as 3 spectroscopic masks, each containing about 30 slits; two of them were centered on the main cluster 
Table 1. Observation summary, cD center (J2000): $11 \mathrm{~h} 31 \mathrm{~m}$ $54.1 \mathrm{~s},-19 \mathrm{~d} 55^{\prime} 40^{\prime \prime}$

\begin{tabular}{lcc}
\hline \hline & $10^{\prime} \times 10^{\prime}$ field center & Time (min) \\
\hline Photometry & $\mathrm{cD}$ & 15,10 \\
$R$ images & $\mathrm{cD}$ & $2 \times 25$ \\
$B$ images & & \\
Spectroscopy & $\mathrm{cD}$ & 45,40 \\
1st mask & $\mathrm{cD}$ & 75,50 \\
2nd mask & $3^{\prime}$ north of the $\mathrm{cD}$ & 60,75 \\
3rd mask &
\end{tabular}

body and the third one on the northern X-ray sub-clump. Observations are summarized in Table 1.

The focal reducer MOS/SIS together with CCD Lick2 $(2048 \times 2048$ pixels of $15 \mu \mathrm{m})$ were used during the run. This CCD is a thick device having a quantum efficiency of $\sim 10 \%$ in the blue. The observing configuration provides a pixel size of $0.314^{\prime \prime}$ over a field of view of about $10^{\prime} \times 10^{\prime}$. The overall image quality was good (stellar FWHM $\sim 0.9^{\prime \prime}$ ) although some optical distortions were conspicuous near the edges of the images due to the optics of the focal reducer. A control problem in the reading of the CCD, namely the random addition of null pixel values, was unfortunately discovered after the A1300 observations. The resulting main effect on the data is to mimic a misalignement between the grism and the CCD columns such that the dispersion direction appears tilted with respect to the CCD columns whereas the image of the slits remain perfectly aligned along the rows; this can be easily corrected, however, the final spectroscopic resolution in somewhat altered.

\section{Photometry}

Detailed inspection of images showed that the cluster central galaxy, a cD, has a double nucleus (Fig. 1) but did not reveal any gravitationally lensed feature, despite the large cluster X-ray luminosity. The $B$ and $R$ frames were prepared using standard pre-reduction techniques and in order to avoid strong vignetting effects, only the $7.1^{\prime} \times 6.5^{\prime}$ inner field was processed. The photometric analysis was performed by means of the SExtractor package (Bertin \& Arnouts 1996): for the $R$ frame (and $B$, respectively), images are first slightly smoothed by a 1 (1.2) pixel $\sigma$ Gaussian (comparable to that of the seeing), then the background is estimated using a $64 \times 64$ pixel mesh. Sources are detected at a 3 (1.2) $\sigma$ level above the local background if having at least 5 pixels at this threshold $(B$ exposures are significantly less deep than the $R$ ones, because of the low CCD efficiency in the blue range). Multiple images are automatically deblended by a multithresholding algorithm and each individual object is finally attributed a "class", a number ranging from 0

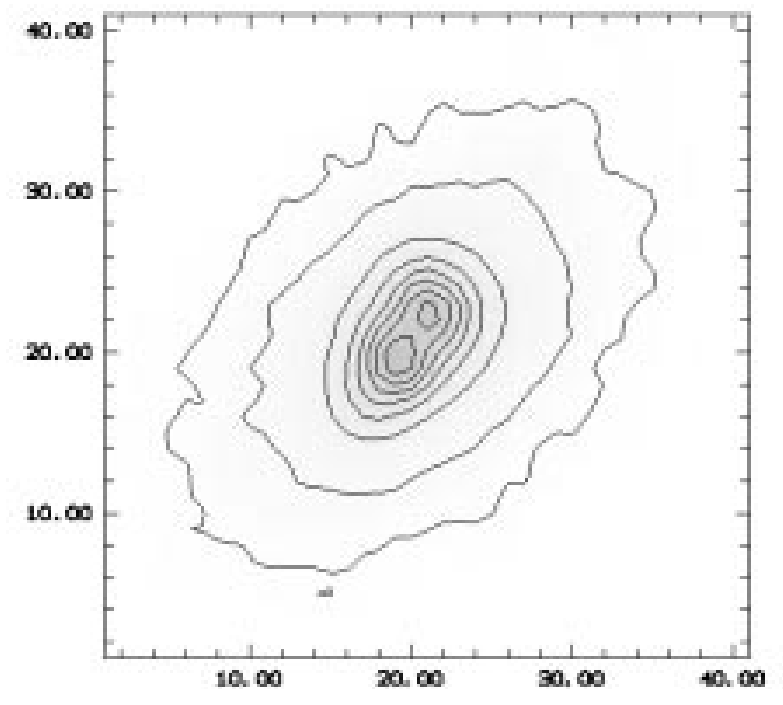

Fig. 1. CFHT R image of A1300 central galaxy revealing a double nucleus. Pixelsize: $0.314^{\prime \prime}$

(galaxy) to 1 (star) by mean of a morphological analysis based on a neuron network. The SExtractor neuron network is of supervised type, the training being done with images containing both stars and galaxies (the input parameters are 8 isophotal areas, the central peak instensity and the seeing). Adaptative aperture magnitudes are computed (inspired from Kron's first moment method); this allows the measurement up to $94 \%$ of the object flux over the almost entire magnitude range. For multiple objects, corrected isophotal magnitudes are used which requires conservation of the total flux for the blend. Since only two frames were available in each colour and that we do not search for very faint objects, each frame was processed individually and magnitudes were averaged at the end of the procedure, providing at the same time estimates for the internal measurement errors in each colour (Tables $2 \& 3$ ).

Table 2. Standard deviation of magnitude differences measured between two frames. In order to estimate errors for objects listed in Table 3, following values have to be divided by $\sqrt{2}$

\begin{tabular}{cc|cc}
\hline \hline$R \leq 20$ & 0.02 & $B \leq 22$ & 0.06 \\
$20 \leq R \leq 20.5$ & 0.03 & $22 \leq B \leq 22.75$ & 0.11 \\
$20.5 \leq R \leq 21.5$ & 0.06 & $22.75 \leq B \leq 23.5$ & 0.14 \\
$21.5 \leq R$ & 0.07 & $23.5 \leq B$ & 0.20 \\
\hline \hline
\end{tabular}

The final photometric calibrations were done using the CCD Sequence in M 92 (Christian et al. 1995) also observed during the same run; for this purpose, stars VCS1, A, B (probably variable) had to be removed 


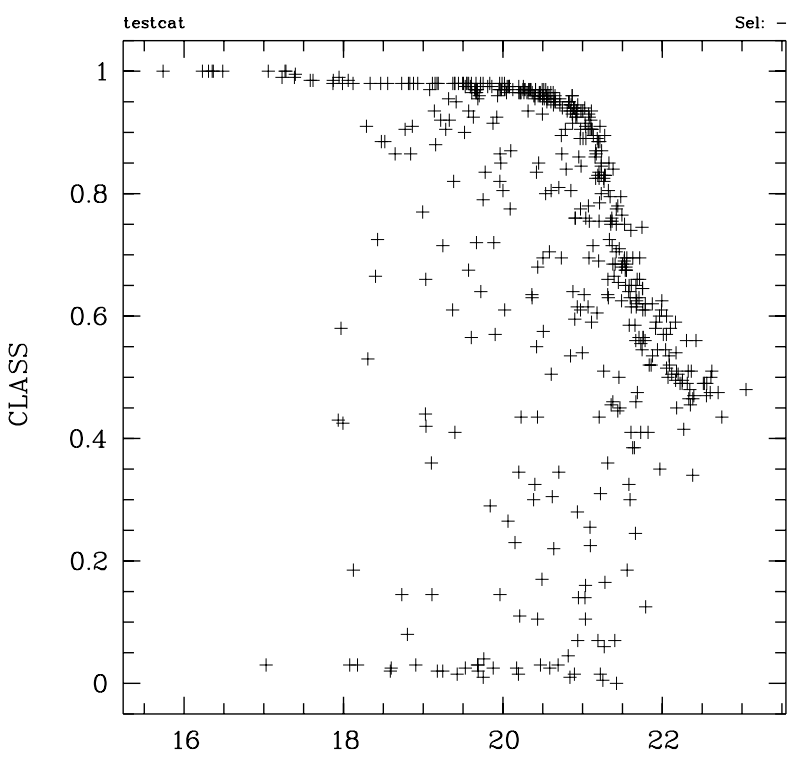

Fig. 2. Galaxy/star separation as a function of $R$ magnitude. Galaxies can be unambiguously identified up to $R \sim 21$. (class $\leq 0.98)$

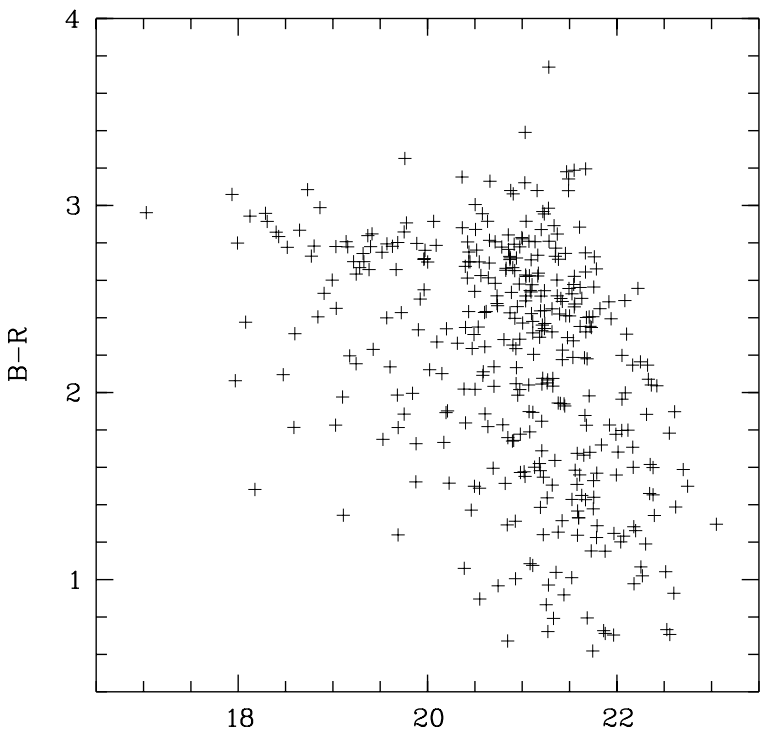

Fig. 3. Colour-magnitude diagramme for objects classified as galaxies

because of obvious inconsistancies. The fraction of galactic stars included in the catalogue was estimated by Robin's model (1995). Between $17 \leq R \leq 21,49$ stars are expected and this allows us to assume that detected objects having a class up to 0.98 may be considered as galaxies within this magnitude range (Fig. 2).

Consequently, only objects having a $R$ class $\leq 0.98$ and detected in all $B$ and $R$ frames are finally retained as galaxies. The rigde observed at the faint magnitude end is a normal feature of the method (cf. Bertin \& Arnouts
Table 3. Photometric analysis of A1300 (beginning; the complete table is available in electronic form at CDS)

\begin{tabular}{|l|l|l|l|c|}
\hline \hline$x$ & $y$ & $m_{R}$ & $B-R$ & $\operatorname{Class}_{R}$ \\
\hline-701.8 & 524.6 & 19.08 & 2.00 & 0.97 \\
-701.3 & 508.2 & 21.42 & 1.31 & 0.71 \\
-697.6 & 326.5 & 19.38 & 2.66 & 0.82 \\
\hline \hline
\end{tabular}

Columns $1 \&$ 2: $x, y$ distance from the field center (cD) in pixel unit $\left(0.314^{\prime \prime}\right)$.

Column 3: $R$ magnitude (average between 2 frames)

Column 4: $B-R$ colour (average between 4 frames)

Column 5: morphological class in the $\mathrm{R}$ band (see text).

1996): it corresponds to the degeneracy of the stellar index to intermediate values as magnitude increases. The fact that it is slightly more prononced here comes from small focus variations across the MOS field of view. Whether faint misclassified stars may still be contained in the final catalogue should not be a problem, at least for the shape of the galaxy luminosity and galaxy number density contours, displayed in Figs. 4 and 5 respectively.

The complete object catalogue is presented in Table 3 and photometric errors given in Table 2. Completness limits are $B=23$ and $R=21$, which corresponds to 180 galaxies. The total catalogue of Table 3 comprises 480 objects, out of these 431 are classified as galaxies.

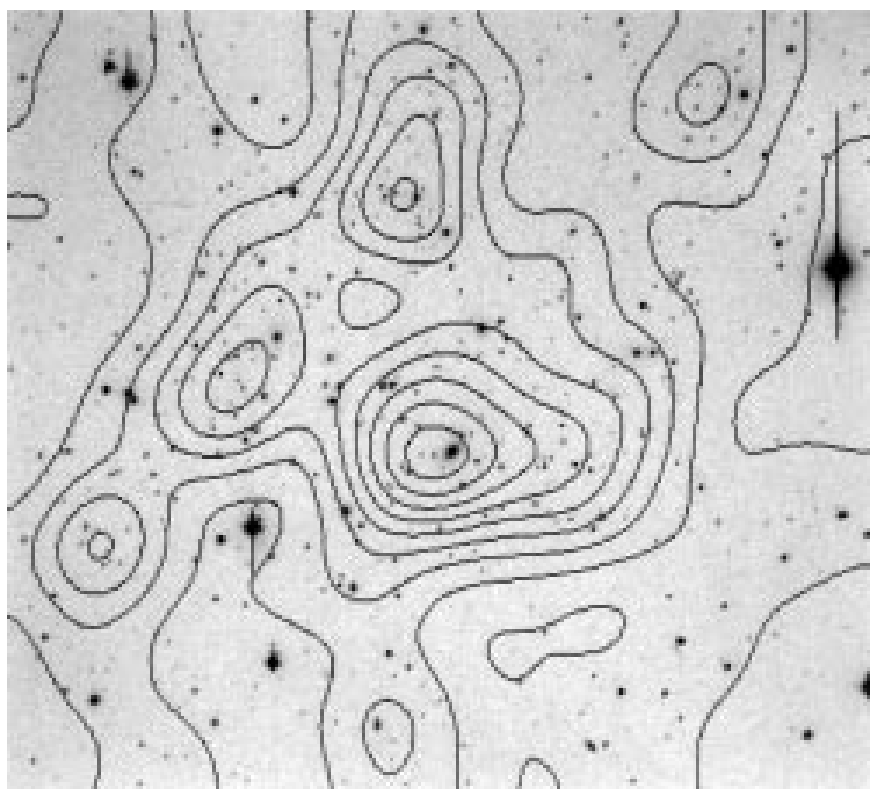

Fig. 4. Galaxy number counts including all galaxies of Table 3. The filtering function has a FWHM of $55^{\prime \prime}$. The contour increment is $\sim 4.5$ galaxies $/ \operatorname{arcmin}^{2}$ (image size $7.14^{\prime} \times 6.54^{\prime}$ ) 


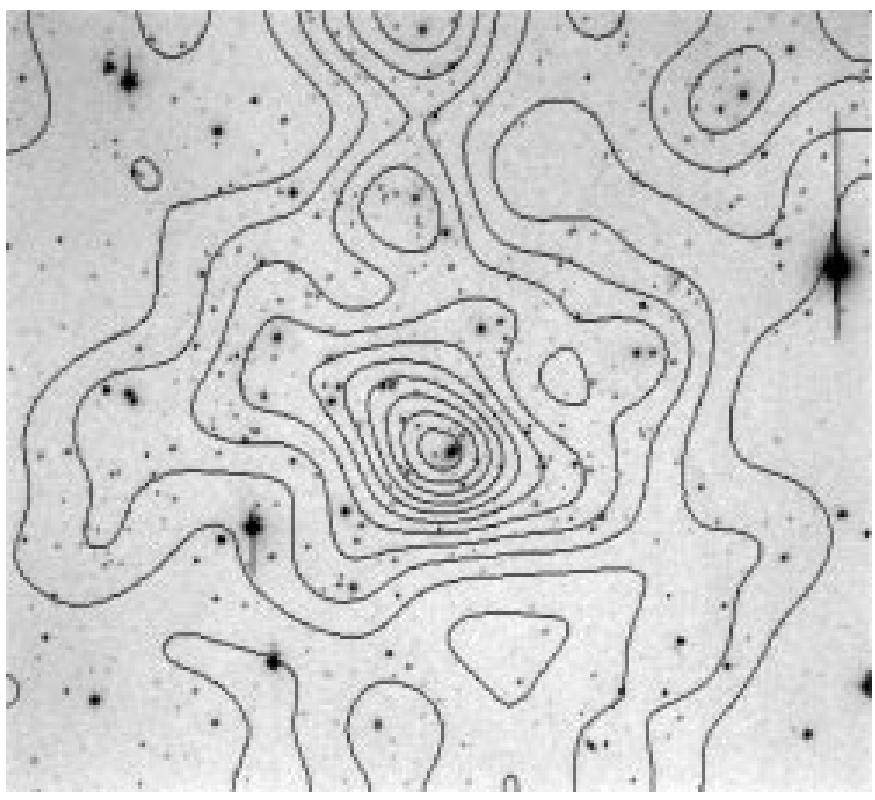

Fig. 5. Galaxy luminosity density coutours including all galaxies of Table 3. The filtering function has a FWHM of $55^{\prime \prime}$. Contour increment is constant in flux. First and last contours correspond respectively to 31.8 and $26.0 \mathrm{~m}_{R} / \mathrm{arcmin}^{2}$ (image size $\left.7.14^{\prime} \times 6.54^{\prime}\right)$

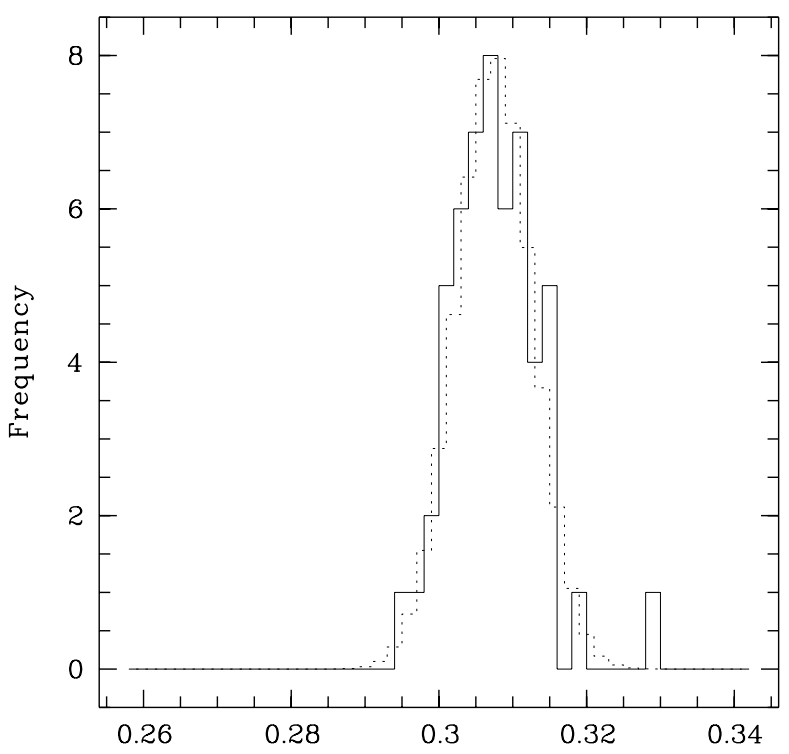

Fig. 6. Cluster galaxy redshift histogramme (binsize: $\delta z=0.002)$; the mean redshift is 0.3072 and the velocity dispersion $1580 \mathrm{~km} / \mathrm{s}$. The dotted line represents the best Gaussian fit

\section{Spectroscopy}

In spectroscopic mode the grism O300 was used. It has a zero deviation at $5900 \AA$, covers approximately 4700 $7900 \AA$ and in the MOS configuration presents a dispersion of $3.59 \AA /$ pixel $\left(0.314^{\prime \prime}\right)$. The slit had a width of $2^{\prime \prime}$, i.e. 6.4 pixels, yielding a resolution of $\sim 23 \AA$ and slightly oversampled spectra which allowed rebinning along the dispersion direction while CCD reading. Typical line features are spread over more than 3 pixels which for bright spectra enables a redshift accuracy of $150 \mathrm{~km} / \mathrm{s}$ and of the order of $300 \mathrm{~km} / \mathrm{s}$ for the faintest objects. Wavelength calibrations were done using internal $\mathrm{He}+$ Ar lamps and after standard reductions (including geometrical rectification of the spectra, see Sect. 1). Redshifts were measured by a cross-correlation method following Tonry \& Davis (1979) and implemented in the MIDAS environment. Errors were estimated as a function of the correlation parameter $r$ : $\delta z=k /(1+r)$, where $k$ has been determined for the present observing configuration by noise degradation of high $\mathrm{S} / \mathrm{N}$ spectra $(k=0.0029)$. The results from the crosscorrelation analysis for faint spectra were in turn checked by hand and the presence of a few conspicuous absorption lines was required, for them to be included in the final list which is presented in Table 4. (Correction for heliocentric motion is $\leq-20 \mathrm{~km} / \mathrm{s}$, and thus totally negligible). Finding charts for the measured galaxies can be found in Figs. 7 and 8.

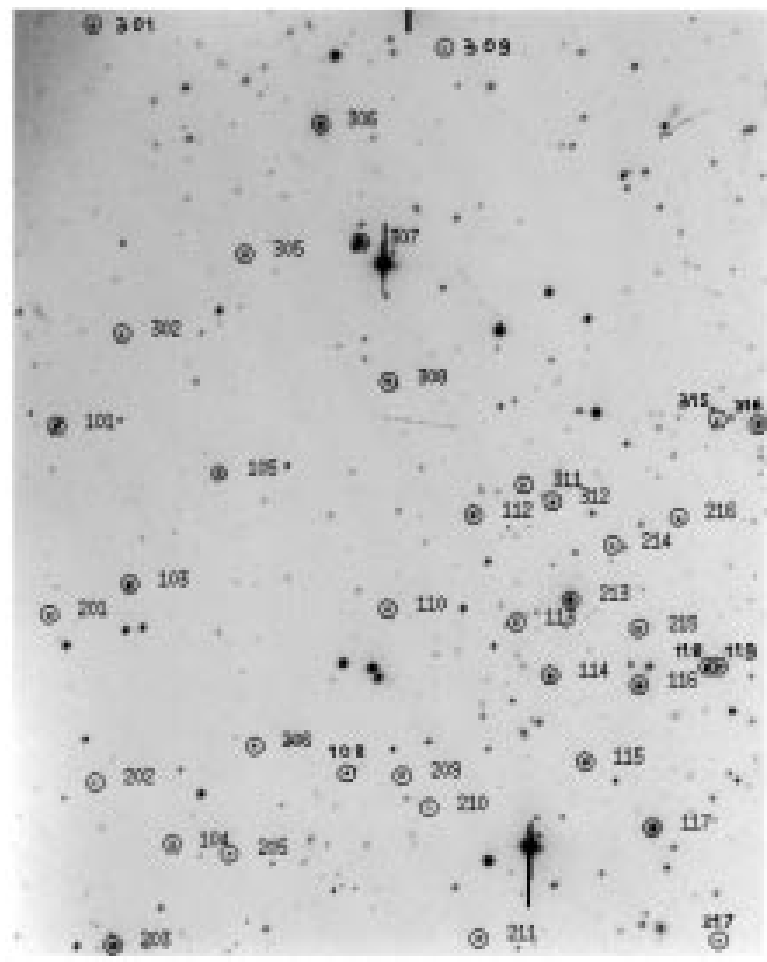

Fig. 7. Finding chart for galaxies with measured redshifts: EAST (image size $4.72^{\prime} \times 6.02^{\prime}$ ) 
Table 4. Spectroscopic analysis of A1300 (also available in electronic form at CDS)

\begin{tabular}{|c|c|c|c|c|c|c|c|c|}
\hline$\overline{~ I D ~}$ & $\overline{\mathrm{RA}_{2000}}$ & $\overline{\text { Dec }_{2000}}$ & $z$ & $\overline{\overline{\delta z} z}$ & $\overline{\bar{Q}}$ & $\overline{\bar{R}}$ & $\overline{\overline{B-R}}$ & "member \\
\hline $101^{\star}$ & 173.0569 & -19.8929 & 0.1954 & 0.0008 & 9 & & & $\mathrm{~N}$ \\
\hline $103^{\star}$ & 173.0488 & -19.9095 & 0.1963 & 0.0013 & 9 & & & $\mathrm{~N}$ \\
\hline 104 & 173.0440 & -19.9371 & 0.3041 & 0.0009 & 1 & & & $\mathrm{Y}$ \\
\hline 105 & 173.0390 & -19.8978 & 0.3150 & 0.0005 & 1 & 19.17 & 2.98 & $\mathrm{Y}$ \\
\hline 108 & 173.0248 & -19.9295 & 0.2256 & 0.0001 & 9 & 19.67 & 2.07 & $\mathrm{~N}$ \\
\hline 110 & 173.0203 & -19.9122 & 0.3032 & 0.0009 & 2 & 19.67 & 1.08 & $\mathrm{Y}$ \\
\hline 112 & 173.0109 & -19.9022 & 0.3067 & 0.0006 & 1 & 19.14 & 2.81 & $\mathrm{Y}$ \\
\hline 113 & 173.0061 & -19.9136 & 0.3017 & 0.0008 & 1 & 19.49 & 2.68 & Y \\
\hline 114 & 173.0024 & -19.9192 & 0.3033 & 0.0004 & 1 & 18.77 & 2.73 & $\mathrm{Y}$ \\
\hline $115^{\star}$ & 172.9985 & -19.9283 & 0.3044 & 0.0011 & 1 & 19.10 & 1.98 & $\mathrm{Y}$ \\
\hline 116 & 172.9926 & -19.9202 & 0.3193 & 0.0009 & 1 & 18.52 & 2.78 & $\mathrm{Y}$ \\
\hline 117 & 172.9911 & -19.9353 & 0.3137 & 0.0004 & 1 & 18.08 & 2.38 & $\mathrm{Y}$ \\
\hline 118 & 172.9850 & -19.9183 & 0.1506 & 0.0006 & 1 & 18.47 & 2.09 & $\mathrm{~N}$ \\
\hline 119 & 172.9839 & -19.9183 & 0.3032 & 0.0007 & 1 & 18.73 & 3.08 & $\mathrm{Y}$ \\
\hline 121 & 172.9754 & -19.9278 & 0.3077 & 0.0007 & 1 & 17.03 & 2.96 & Y \\
\hline 122 & 172.9706 & -19.9108 & 0.3159 & 0.0008 & 1 & 18.12 & 2.94 & Y \\
\hline 124 & 172.9634 & -19.9352 & 0.3002 & 0.0009 & 1 & 19.65 & 2.59 & $\mathrm{Y}$ \\
\hline 127 & 172.9483 & -19.9148 & 0.3014 & 0.0007 & 1 & 18.29 & 2.96 & $\mathrm{Y}$ \\
\hline 128 & 172.9459 & -19.9148 & 0.3027 & 0.0007 & 1 & 18.86 & 2.99 & Y \\
\hline 132 & 172.9278 & -19.9436 & 0.3044 & 0.0008 & 1 & 18.65 & 2.87 & Y \\
\hline 133 & 172.9233 & -19.9099 & 0.3150 & 0.0010 & 1 & 19.84 & 1.99 & $\mathrm{Y}$ \\
\hline 134 & 172.9194 & -19.9378 & 0.3076 & 0.0014 & 9 & & & $\mathrm{Y}$ \\
\hline 201 & 173.0576 & -19.9128 & 0.3146 & 0.0007 & 1 & & & Y \\
\hline 202 & 173.0525 & -19.9305 & 0.3048 & 0.0011 & 2 & & & Y \\
\hline 203 & 173.0504 & -19.9477 & 0.3063 & 0.0005 & 1 & & & Y \\
\hline $205^{\star}$ & 173.0377 & -19.9380 & 0.3121 & 0.0011 & 1 & 20.02 & 2.12 & $\mathrm{Y}$ \\
\hline 206 & 173.0349 & -19.9266 & 0.3087 & 0.0009 & 1 & 19.92 & 2.50 & $\mathrm{Y}$ \\
\hline 209 & 173.0187 & -19.9298 & 0.3045 & 0.0007 & 1 & 19.60 & 2.77 & Y \\
\hline 210 & 173.0157 & -19.9331 & 0.3101 & 0.0012 & 2 & & & Y \\
\hline 211 & 173.0102 & -19.9470 & 0.2947 & 0.0010 & 2 & 19.60 & 2.14 & Y \\
\hline 213 & 173.0002 & -19.9112 & 0.3016 & 0.0009 & 9 & 18.03 & 2.85 & $\mathrm{Y}$ \\
\hline 214 & 172.9956 & -19.9054 & 0.3109 & 0.0009 & 1 & 20.02 & 2.79 & $\mathrm{Y}$ \\
\hline 215 & 172.9926 & -19.9142 & 0.3115 & 0.0010 & 9 & 19.03 & 2.45 & Y \\
\hline 216 & 172.9884 & -19.9024 & 0.3121 & 0.0008 & 9 & 19.55 & 2.43 & Y \\
\hline 217 & 172.9837 & -19.9472 & 0.3137 & 0.0008 & 1 & 20.00 & 2.70 & Y \\
\hline 219 & 172.9753 & -19.9277 & 0.3077 & 0.0007 & 2 & & & Y \\
\hline 220 & 172.9717 & -19.9289 & 0.3021 & 0.0009 & 1 & 19.53 & 2.79 & Y \\
\hline 223 & 172.9566 & -19.9096 & 0.3018 & 0.0008 & 1 & 19.32 & 2.74 & Y \\
\hline $225^{\star}$ & 172.9477 & -19.9263 & 0.3031 & 0.0009 & 1 & 20.08 & 2.73 & $\mathrm{Y}$ \\
\hline 229 & 172.9272 & -19.9033 & 0.2989 & 0.0010 & 1 & 19.63 & 2.78 & $\mathrm{Y}$ \\
\hline 301 & 173.0530 & -19.8504 & 0.3598 & 0.0012 & 2 & & & $\mathrm{~N}$ \\
\hline 302 & 173.0497 & -19.8830 & 0.0588 & 0.0010 & 2 & & & $\mathrm{~N}$ \\
\hline 305 & 173.0362 & -19.8746 & 0.3098 & 0.0007 & 1 & 19.08 & 1.99 & Y \\
\hline 306 & 173.0279 & -19.8609 & 0.3115 & 0.0010 & 1 & & & $\mathrm{Y}$ \\
\hline 307 & 173.0235 & -19.8734 & 0.2063 & 0.0010 & 1 & & & $\mathrm{~N}$ \\
\hline 308 & 173.0203 & -19.8881 & 0.3044 & 0.0009 & 1 & 19.16 & 2.78 & $\mathrm{Y}$ \\
\hline 309 & 173.0142 & -19.8529 & 0.3108 & 0.0009 & 1 & & & Y \\
\hline 311 & 173.0054 & -19.8990 & 0.3040 & 0.0010 & 1 & 19.70 & 2.68 & Y \\
\hline 312 & 173.0022 & -19.9008 & 0.3437 & 0.0013 & 2 & 19.33 & 2.70 & $\mathrm{~N}$ \\
\hline 315 & 172.9841 & -19.8922 & 0.3108 & 0.0006 & 1 & 19.66 & 2.49 & $\mathrm{Y}$ \\
\hline 316 & 172.9797 & -19.8927 & 0.3082 & 0.0006 & 1 & 18.40 & 2.86 & Y \\
\hline 317 & 172.9754 & -19.8976 & 0.3076 & 0.0008 & 1 & 18.43 & 2.83 & $\mathrm{Y}$ \\
\hline 318 & 172.9704 & -19.8795 & 0.3108 & 0.0011 & 1 & & & Y \\
\hline
\end{tabular}


Table 4. continued

\begin{tabular}{|l|l|l|l|l|l|l|l|c|}
\hline \hline $\mathrm{ID}$ & $\mathrm{RA}_{2000}$ & $\mathrm{Dec}_{2000}$ & $z$ & $\delta z$ & $Q$ & $R$ & $B-R$ & member \\
\hline 319 & 172.9671 & -19.9122 & 0.3099 & 0.0010 & 9 & 19.55 & 2.81 & $\mathrm{Y}$ \\
$320^{\star}$ & 172.9595 & -19.8613 & 0.2999 & 0.0013 & 2 & & & $\mathrm{Y}$ \\
$321^{\star}$ & 172.9570 & -19.8978 & 0.2980 & 0.0013 & 9 & 19.42 & 2.23 & $\mathrm{Y}$ \\
324 & 172.9494 & -19.8692 & 0.3082 & 0.0006 & 1 & 19.25 & 2.63 & $\mathrm{Y}$ \\
325 & 172.9462 & -19.8808 & 0.3150 & 0.0012 & 2 & & & $\mathrm{Y}$ \\
326 & 172.9401 & -19.8857 & 0.3283 & 0.0009 & 9 & & & $\mathrm{~N}$ \\
327 & 172.9352 & -19.8818 & 0.2571 & 0.0009 & 1 & 19.22 & 2.70 & $\mathrm{~N}$ \\
328 & 172.9318 & -19.8797 & 0.2575 & 0.0007 & 1 & 17.99 & 2.80 & $\mathrm{~N}$ \\
329 & 172.9250 & -19.8737 & 0.2569 & 0.0008 & 1 & 18.80 & 2.78 & $\mathrm{~N}$ \\
332 & 172.9128 & -19.8685 & 0.3100 & 0.0010 & 2 & & & $\mathrm{Y}$ \\
333 & 172.9078 & -19.8611 & 0.3079 & 0.0009 & 1 & & & $\mathrm{Y}$ \\
\hline \hline
\end{tabular}

Column 1: internal number refering to Figs. 7, 8.

Columns 2 \& 3: RA and Dec (J2000), decimal degrees. Galaxy positions were interactively measured on the STScI digitized blue sky survey images having a pixel size of $1.7^{\prime \prime}$, and should have an accuracy of $\sim 3.4^{\prime \prime}$.

Column 4: redshift.

Column 5: error on the redshift.

Column 6: $z$ measurement quality (1: highest peak in the correlation function, 2: 2nd peak, 9: interactive measurement with lines).

Column 7: $R$ magnitude (only for galaxies within the area processed for photometry).

Column 8: $B-R$ index.

Column 9: cluster member galaxy ( $3 \sigma$ clipping method).

Presence of emission lines $(*)$ :

101: $\mathrm{H}_{\alpha}$.

103: $\mathrm{H}_{\alpha}$.

115: [OII] at 3727, $\mathrm{H}_{\beta},[\mathrm{OIII}]$ at 4959 and 5007, OI at 6300 and $6364, \mathrm{H}_{\alpha}$, NII at 6549 and 6583 , SII at 6717 and 6734 .

205: $\mathrm{H}_{\alpha}, \mathrm{H}_{\beta}$ ?

225: OI at 6300 and 6364 .

320: $\mathrm{H}_{\alpha}$ ?

321: $\mathrm{H}_{\alpha}$ ? SII at 6717.

The two nuclei of the $\mathrm{cD}$ galaxy correspond to numbers $121 \& 219$ and show totally similar redshifts at the present spectrocsopic resolution. 